\title{
Modified Projective Synchronization of Chaotic Systems with Noise Disturbance, an Active Nonlinear Control Method
}

\author{
Hamed Tirandaz, Hamidreza Tavakoli and Mohsen Ahmadnia \\ Electrical and Computer Engineering Faculty, Hakim Sabzevari University, Sabzevar, Iran
}

\begin{tabular}{|c|c|}
\hline Article Info & ABSTRACT \\
\hline $\begin{array}{l}\text { Article history: } \\
\text { Received: Jan 15, } 2017 \\
\text { Revised: Jul 25, 2017 } \\
\text { Accepted: Aug 9, } 2017\end{array}$ & $\begin{array}{l}\text { The synchronization problem of chaotic systems using active modified projective non- } \\
\text { linear control method is rarely addressed. Thus the concentration of this study is to } \\
\text { derive a modified projective controller to synchronize the two chaotic systems. Since, } \\
\text { the parameter of the master and follower systems are considered known, so active } \\
\text { methods are employed instead of adaptive methods. The validity of the proposed con- }\end{array}$ \\
\hline $\begin{array}{l}\text { Keyword: } \\
\text { Active method } \\
\text { Nonlinear control } \\
\text { Modified Projective } \\
\text { synchronization (MPS) }\end{array}$ & $\begin{array}{l}\text { troller is studied by means of the Lyapunov stability theorem. Furthermore, some } \\
\text { numerical simulations are shown to verify the validity of the theoretical discussions. } \\
\text { The results demonstrate the effectiveness of the proposed method in both speed and } \\
\text { accuracy points of views. }\end{array}$ \\
\hline Stability theorem & $\begin{array}{r}\text { Copyright (C) } 2017 \text { Institute of Advanced Engineering and Science. } \\
\text { All rights reserved. }\end{array}$ \\
\hline \multicolumn{2}{|c|}{$\begin{array}{l}\text { Corresponding Author: } \\
\text { Hamed Tirandaz } \\
\text { Hakim Sabzevari University } \\
\text { Electrical and Computer Engineering Faculty, Hakim Sabzevari University, Sabzevar, Iran. } \\
\text { Phone +98051-4401288 } \\
\text { Email: tirandaz@ } \text { hsu.ac.ir }\end{array}$} \\
\hline
\end{tabular}

\section{INTRODUCTION}

Master-slave synchronization of chaotic systems is strikely nonlinear, since the aperiodic and nonregular behavior of chaotic systems and their sensitivity to the initial conditions. Chaotic behavior may appear in many physical systems. So, chaos synchronization subject has received a great deal of attention in the last to decades, due to its potential applications in physics, chemistry, electrical engineering, secure communication and so on[1]. Up to now, many types of controling methods are revealed and investigated for control and synchronization of chaotic systems. Active method[2, 3, 4, 5, 6], adaptive method [7, 8, 9], linear feedback method $[10,11]$, nonlinear feedback method [12, 14, 15], sliding mode method [16, 17, 18], impulsive method [19], phase method [20], generalized method [21], robust synchronization [13] and projective method [22, 23, 24] are some of the introduced methods by the researchers. Among these methods, synchronization with some types of projective methods are extensively investigated in the last decades, since the faster synchronization due to its synchronization scaling factors, which master and slave chaotic systems would be synchronized up to a proportional rate. Projective lag method [25], modified projective synchronization (MPS) [26, 27, 28], function projective synchronization (FPS)[29], modified function projective synchronization [30, 28], generalized function projective synchronization [31, 32] and modified projective lag synchronization[33, 34] are some generalized schemes of projective method, which utilize some type of scaling factors.

When the parameters of a chaotic system are known beforehand, active related methods are preferably chosen than adaptive methods. Active synchronization problem of two chaotic systems with known parameters are vastly investigated by the researchers. For example, in [5, 3, 35], the active controlling method is studied for synchronization of two typical chaotic systems. And also, in [2], an active method for controling the behavior of a unified chaotic system is presented. Chaos synchronization of complex Chen and Lu chaotic systems are addressed in citeMahmoud, with designing an active control method. Furthermore, in [36] active 
synchronization of two different fractional order chaotic system is studied.

Consequently, the modified projective synchronization of two chaotic systems with known system parameters by acitve control method are rarely investigated by the researchers. Therefore, in the present study, the modified projective synchronization problem is achieved by means of active nonlinear control method. An appropriate feedback controller is designed to control the behavior the state variables of the follower system to track the trajectories of the leader system state variables. In Section 2, the problem of chaos synchronization is discussed. In addition, the validity of the proposed synchronization method is verified by means of Lyapunov stability theorem. Then, in Section 3, some experiments are derived to show the effectiveness of the proposed method. Moreover, some simulations are carried out. Finally, some concluding remarks are given in Section 4.

\section{SYNCHRONIZATION}

A wide variety of chaotic systems can be represented as follows:

$$
\dot{\boldsymbol{x}}=f(\boldsymbol{x}) \boldsymbol{\Phi}+F(\boldsymbol{x})+\boldsymbol{\eta}
$$

Where $\mathbf{x}=\left(x_{1}, x_{2}, \cdots, x_{n}\right)^{T}$ is the state variables vector of the system (1). $\mathbf{\Phi}=\left(\phi_{1}, \phi_{2}, \cdots, \phi_{n}\right)^{T} \in \mathbf{R}^{n \times 1}$ and $\eta=\left(\eta_{1}, \eta_{2}, \cdots, \eta_{n}\right)^{T} \in \mathbf{R}^{n \times 1}$ are two vectors denoting the unknown parameter vector of the system and the external distributive noise of the system, respectively. $f(\boldsymbol{x}) \in \mathbf{R}^{n \times n}$ and $F(\boldsymbol{x}) \in \mathbf{R}^{n \times 1}$ stand for the linear and nonlinear matrix of functions, respectively. Let the dynamical system (1) as the leader system. Then the follower system can be given by another chaotic function as follows:

$$
\dot{\boldsymbol{y}}=g(\boldsymbol{y}) \hat{\boldsymbol{\Phi}}+G(\boldsymbol{y})+\boldsymbol{u}
$$

Where $\boldsymbol{y}=\left(y_{1}, y_{2}, \cdots, y_{n}\right)^{T}$ presents the state variables vector of the follower system $(2) . \hat{\boldsymbol{\Phi}}=\left(\hat{\phi}_{1}, \hat{\phi}_{2}, \cdots, \hat{\phi}_{n}\right) \in$ $\mathbf{R}^{n \times 1}$ denotes the estimation of leader system parameters vector $\boldsymbol{\Phi}$. Moreover, $g(\boldsymbol{y}) \in \mathbf{R}^{n \times n}$ and $G(\boldsymbol{y}) \in$ $\mathbf{R}^{n \times 1}$ are the linear and nonlinear matrix of functions, respectively. In the proposed active nonlinear control method, an appropriate controller $\boldsymbol{u}$ is designed which the states of leader system (1) are synchronized with their corresponding states at the follower chaotic sytem (2), base on the modified projective synchronization error that is defined as follows:

$$
e=\boldsymbol{y}-\Lambda x
$$

Where $\boldsymbol{\Lambda}=\operatorname{diag}\left\{\lambda_{1}, \lambda_{2}, \cdots, \lambda_{n}\right\}$ represents the modified scaling factors and $\boldsymbol{e}=\left(e_{1}, e_{2}, \cdots, e_{n}\right)^{T} \in \mathbf{R}^{n \times 1}$ stands for synchronization error vector. Then the dynamical synchronizaton error can be obtatined as follows:

$$
\begin{aligned}
\dot{\boldsymbol{e}} & =\dot{\boldsymbol{y}}-\boldsymbol{\Lambda} \dot{\boldsymbol{x}} \\
& =g(\boldsymbol{y}) \boldsymbol{\Phi}+G(\boldsymbol{y})+\boldsymbol{u}-f(\boldsymbol{x}) \boldsymbol{\Phi}-\boldsymbol{\Lambda} F(\boldsymbol{x})-\boldsymbol{\Lambda} \boldsymbol{\eta}
\end{aligned}
$$

Where $\overline{\boldsymbol{\eta}}$ denotes the estimation of noise distrubance $\boldsymbol{\eta}$.

Definition 1. For the leader system (1) and the follower system (2), the chaos synchronization would be achieved if an appropriate control is designed to force the state variables of the follower system to track the trajectories of the leader one, meanly, the synchronization error vector (3) converges to zero, as time goes to infinity,i. e:

$$
\lim _{t \rightarrow \infty}\|e(t)\|=0
$$

which ||.|| denotes 2-norm. Chaos synchronization can be achieved by deriving an appropriate feedback controller, which is the subject of the following theorem.

Theorem 1. The leader system (1) with the state variables vector $\boldsymbol{x}$ and the follower system (2) with the state variables vector $\boldsymbol{y}$, the parameters vector $\boldsymbol{\Phi}$ and any noise disturbance vector $\boldsymbol{\eta}$, would be synchronized for any initial state variables $\boldsymbol{x}(0)$ and $\boldsymbol{y}(0)$, if the active feedback control law is defined as follows:

$$
\boldsymbol{u}=-[g(\boldsymbol{y})-f(\boldsymbol{x})] \boldsymbol{\Phi}-[G(\boldsymbol{y})-\boldsymbol{\Lambda} F(\boldsymbol{x})]+\boldsymbol{\Lambda} \overline{\boldsymbol{\eta}}-\boldsymbol{K} \boldsymbol{e}
$$

Where $\bar{\eta}$ can be estimated dynamically as follows:

$$
\dot{\bar{\eta}}=-\Lambda e-\Psi(\bar{\eta}-\eta)
$$


Where $\boldsymbol{K}=\operatorname{diag}\left\{k_{1}, k_{2}, \cdots, k_{n}\right\}$ and $\boldsymbol{\Psi}=\operatorname{diag}\left\{\psi_{1}, \psi_{2}, \cdots, \psi_{n}\right\}$ are two diagonal matrix with positive values for their main diagonal elements.

Proof. Let the Lyapunov stability function as follows:

$$
V=\frac{1}{2} \boldsymbol{e} e^{T}+\frac{1}{2}(\overline{\boldsymbol{\eta}}-\boldsymbol{\eta})(\overline{\boldsymbol{\eta}}-\boldsymbol{\eta})^{T}
$$

It is obvious that the Lyapunov function defined in (7) is positive definite. With calculating its time derivative, we have:

$$
\dot{V}=\dot{\boldsymbol{e}} \boldsymbol{e}^{T}+\dot{\overline{\boldsymbol{\eta}}}(\overline{\boldsymbol{\eta}}-\boldsymbol{\eta})^{T}
$$

Then, substituting the dynamical representation of synchronization error vector (4) and consequently considering the proposed feedback controller (5) and the noise estimation (6), one can get:

$$
\dot{V}=-\boldsymbol{K} \boldsymbol{e} \boldsymbol{e}^{T}-\boldsymbol{\Psi}(\hat{\boldsymbol{\eta}}-\boldsymbol{\eta})(\hat{\boldsymbol{\eta}}-\boldsymbol{\eta})^{T}
$$

Therefore, derivative of $\mathrm{V}$ is negative definite, when $\boldsymbol{K}$ and $\boldsymbol{\Psi}$ are diagonal matrix with positive elements on their primary diagonal elements. In the following section, some numerical results are given to show the effectiveness of the proposed synchronization method.

\section{NUMERICAL SIMULATIONS}

This section is devoted to the synchronization of two different chaotic or hyperchaotic systems. In the following subsection, chaos synchronization between two chaotic systems, Zhang chaotic system and Lorenz chaotic system is addressed. Then, the synchronizaton problem between two hyperchaotic system as Chen hyperchaotic system and Lorenz hyperchaotic system is studied in the last subsection

\section{1. chaotic systems}

Chaos synchronization between Zhang chaotic system [14] and the Lü chaotic system [37] is addressed in this subsection. The Zhang chaotic system is given by a three simple integer-based and nonlinear differential equations that depends on the three positive real parameters as follows

$$
\begin{aligned}
& \dot{x_{1}}=a\left(x_{2}-x_{1}\right)-x_{2} x_{3} \\
& \dot{x_{2}}=b x_{1}-x_{1}^{2} \\
& \dot{x_{3}}=-c x_{3}+x_{2}^{2}
\end{aligned}
$$

Where $x_{1}, x_{2}$ and $x_{3}$ are the state variables of the system and $\mathrm{a}, \mathrm{b}$, and $\mathrm{c}$ are the three constant parameters of the system. When $a=10, b=30$ and $c=6$, the behaviour of the system is chaotic. The phase portraits of the system is shown in Fig. 1, with initial state variables $x_{1}(0)=5, x_{2}(0)=2$ and $x_{3}(0)=30$.

In addition, the Lü chaotic system can be described as follows:

$$
\begin{aligned}
& \dot{y_{1}}=\alpha_{1}\left(y_{2}-y_{1}\right) \\
& \dot{y_{2}}=\alpha_{2} y_{2}-y_{1} y_{3} \\
& \dot{y_{3}}=y_{1} y_{2}-\alpha_{3} y_{3}
\end{aligned}
$$

Where $y_{1}, y_{2}$ and $y_{3}$ are the state variables of the system and $\alpha_{1}, \alpha_{2}$ and $\alpha_{3}$ are the parameter of the system. The chaotic behavior of the Lü system is shown in Fig. 2, with system parameters as: $\alpha_{1}=2.1, \alpha_{2}=30$ and $\alpha_{3}=0.6$, and state variables initial values as: $x_{1}(0)=4.3, x_{2}(0)=7.2$ and $x_{3}(0)=5.8$.

The Zhang chaotic system (10) can be rewritten based on the leader system (1) as follows:

$$
\begin{aligned}
& \dot{x_{1}}=a\left(x_{2}-x_{1}\right)-x_{2} x_{3}+\eta_{1} \\
& \dot{x_{2}}=b x_{1}-x_{1}^{2}+\eta_{2} \\
& \dot{x_{3}}=-c x_{3}+x_{2}^{2}+\eta_{3}
\end{aligned}
$$



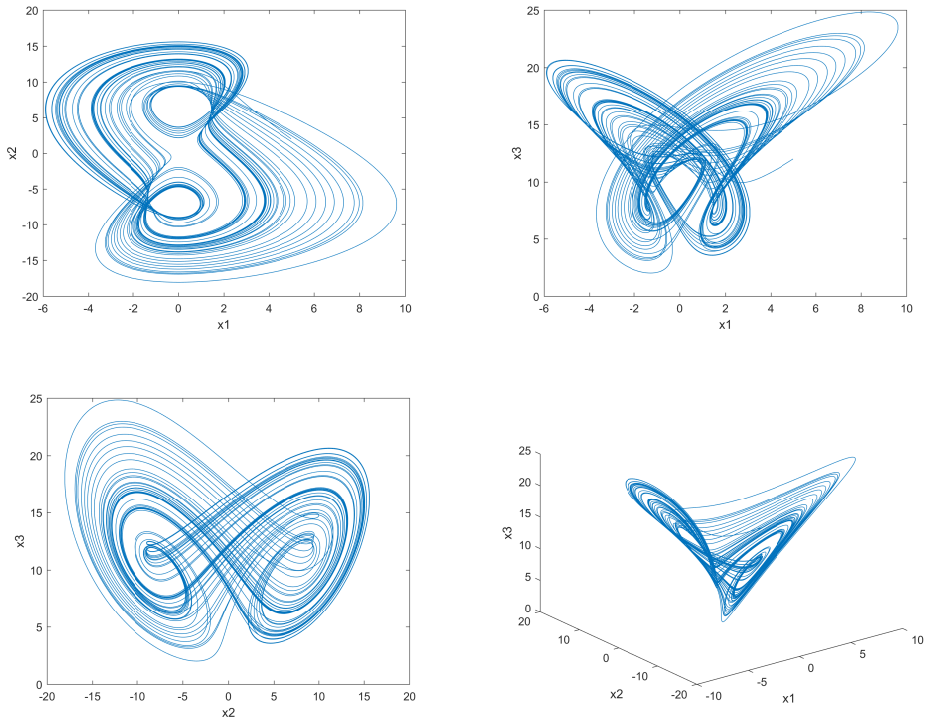

Figure 1. Phase portraits of hte Zhang chaotic system
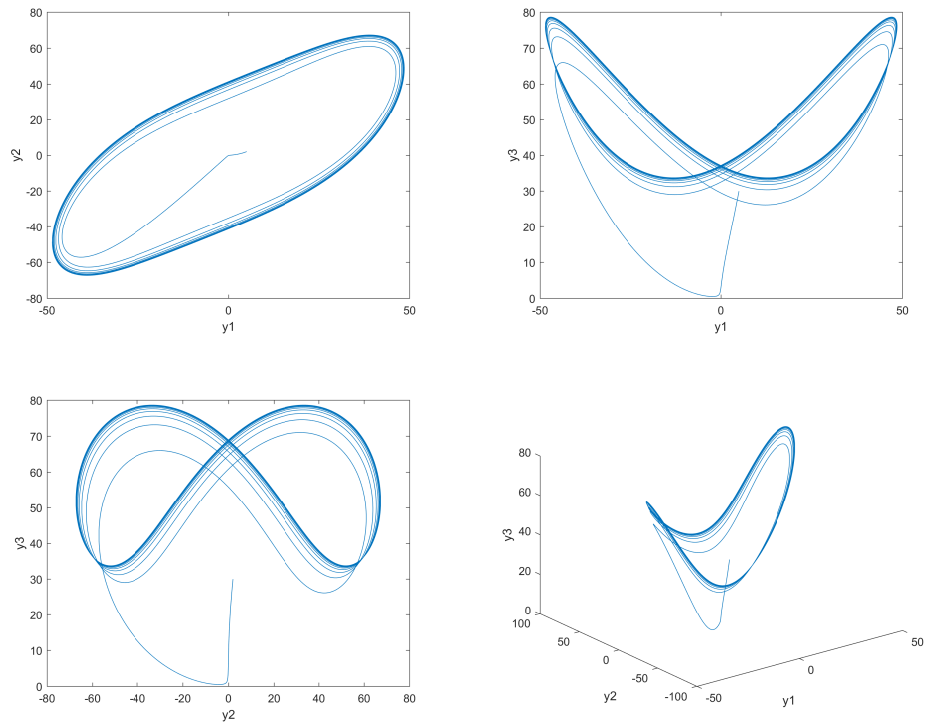

Figure 2. Phase portraits of hte Lu chaotic system 
Where $\eta_{1}, \eta_{2}$ and $\eta_{3}$ are the three noise disturbance corresponding to the state variables $x_{1}, x_{2}$ and $x_{3}$, respectively. Then, the Lü chaotic system (11) can be represented as the follower system as follows:

$$
\begin{aligned}
& \dot{y_{1}}=a\left(y_{2}-y_{1}\right)+u_{1} \\
& \dot{y_{2}}=b y_{2}-y_{1} y_{3}+u_{2} \\
& \dot{y_{3}}=y_{1} y_{2}-c y_{3}+u_{3}
\end{aligned}
$$

According to the proposed control law (5) and noise disturbance estimation (6), we define the following feedback controller as:

$$
\begin{aligned}
& u_{1}=-a y_{2}+\lambda_{1} a x_{1}+a e_{1}-\lambda_{1} x_{2} x_{3}+\lambda_{1} \bar{\eta}_{1}-k_{1} e_{1} \\
& u_{2}=-b y_{2}+y_{1} y_{3}+\lambda_{2}\left(b x_{1}-x_{1}^{2}\right)+\lambda_{2} \bar{\eta}_{2}-k_{2} e_{2} \\
& u_{3}=-y_{1} y_{2}+c e_{3}+\lambda_{3} x_{2}^{2}+\lambda_{3} \bar{\eta}_{3}-k_{3} e_{3},
\end{aligned}
$$

and the noise disturbance estimation as:

$$
\begin{aligned}
& \dot{\bar{\eta}}_{1}=-\lambda_{1} e_{1}-\psi_{1}\left(\bar{\eta}_{1}-\eta_{1}\right) \\
& \dot{\bar{\eta}}_{2}=-\lambda_{2} e_{2}-\psi_{2}\left(\bar{\eta}_{2}-\eta_{2}\right) \\
& \dot{\bar{\eta}}_{3}=-\lambda_{3} e_{3}-\psi_{3}\left(\bar{\eta}_{3}-\eta_{3}\right)
\end{aligned}
$$

Assume the parameter of the Zhang chaotic system as $a=10, b=30$ and $c=6$ and the initial values for the drive chaotic system (12) are taken as, $x_{1}(0)=12, x_{2}(0)=5$, and, $x_{3}(0)=6.5$. In additiion, the initial values of the response $\mathrm{L}$ system $(3)$ are selected as: $y_{1}(0)=2, y_{2}(0)=15$ and $y_{3}(0)=0$. Consider the nosie disturbance values as $\eta_{1}=0.8, \eta_{2}=0.6$ and $\eta_{3}=0.3$ and also their corresponding estimation ititial values as $\bar{\eta}_{1}=0.15, \bar{\eta}_{2}=0.2$ and $\bar{\eta}_{3}=0.1$. Let the gain constants as $k_{1}=2, k_{2}=2, k_{3}=2, \phi_{1}=1.5, \phi_{2}=1.5$ and $\phi_{3}=1.5$.

The validify of the proposed synchronization method for contorling the behavior of the Lu chaotic system (13) to track the motion trajectories of the Zhang chaotic system (12) and the noise disturbance estimation are shown in Figure 3 and 4, respectively. Figure 3 shows that the state variables of the system (13) track effectively the motion trajectories of the leader chaotic system. In addition, in Figure 4 exhibit that the distance between noise disturbance and its estimation values converge to zero.
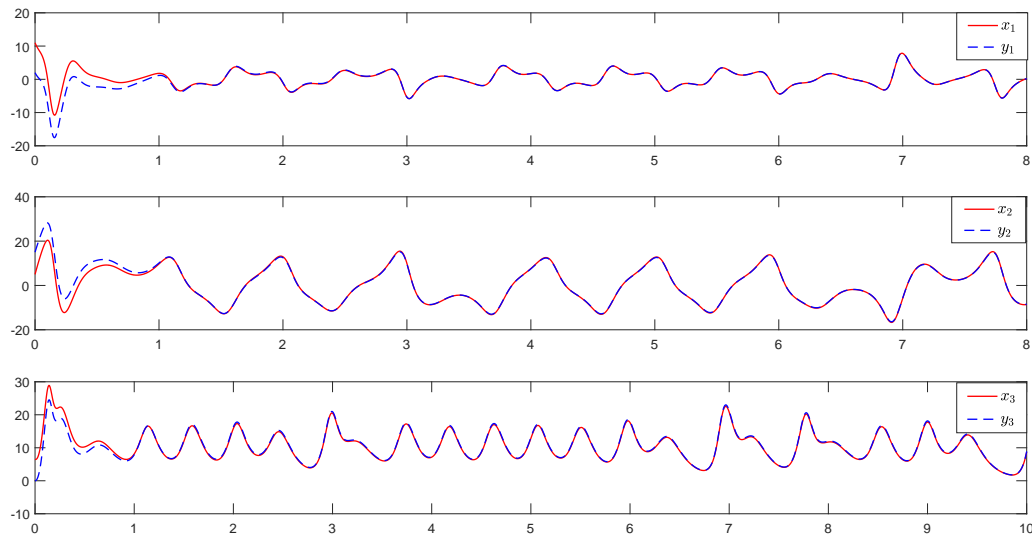

Figure 3. Time responce of the drive Zhang chaotic system and the response Lorenz chaotic system

\subsection{Hyperchaotic systems}

In this subsection, the synchronization between two hyperchaotic systems as Chen hyperchaotc system and Lorenz hyperchaotic system is investivated via the proposed control method. The Chen hyperchaotic 


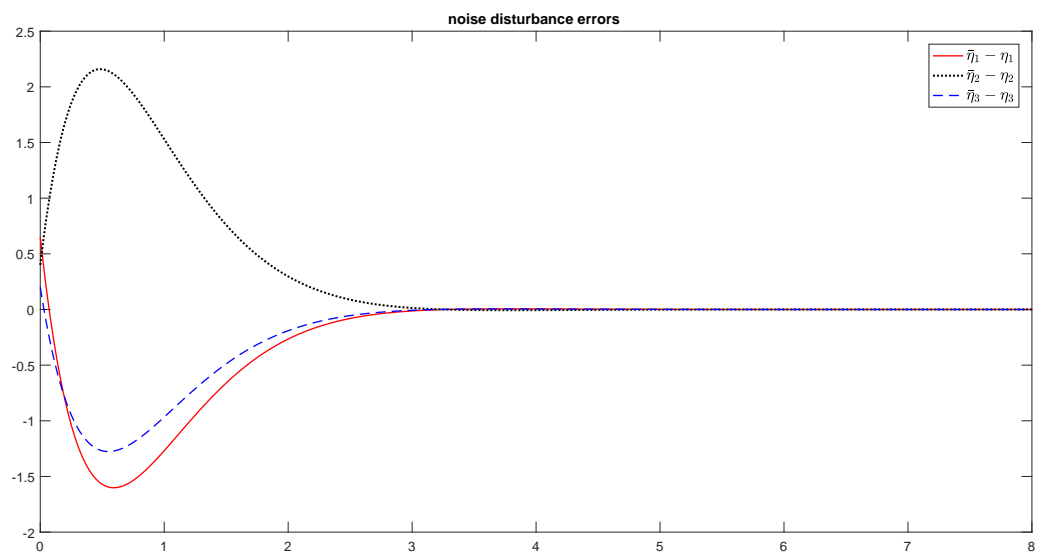

Figure 4. Time responce of the noise disturbance estimation

system is introduced in [38], as an extention of a three-dimensional Chen chaotic system as follows:

$$
\begin{aligned}
& x_{1}=a\left(x_{2}-x_{1}\right)+x_{4} \\
& x_{2}=d x_{1}+c x_{2}-x_{1} x_{3} \\
& x_{3}=x_{1} x_{2}-b x_{3} \\
& x_{4}=x_{1} x_{2}+r x_{4}
\end{aligned}
$$

Where $x_{1}, x_{2}, x_{3}$ and $x_{4}$ are the state variables and $a, b, c$ and $d$ are the parameter of the system. The phase prortait of the system (16) is shown in Fig. 5, with state variables $x_{1}(0)=, x_{2}(0)=, x_{3}(0)=$ and $x_{4}(0)=$ and the parameters as $a=35, b=3, c=12, d=7$ and $\mathrm{r}=0.5$. As it can be seen the behavior of the system (16) is hyperchaotic. The Lorenz hyperchaotic system, which was introduced in [39], can be described as follows:

$$
\begin{aligned}
& y_{1}=\alpha_{1}\left(y_{2}-y_{1}\right)+y_{4} \\
& y_{2}=-y_{1} y_{3}+\alpha_{3} y_{1}-y_{2} \\
& y_{3}=y_{1} y_{2}-\alpha_{2} y_{3} \\
& y_{4}=-y_{1} y_{3}+\alpha_{4} y_{4}
\end{aligned}
$$

Where $y_{1}, y_{2}, y_{3}$ and $y_{4}$ are the state variabels, $a, b, c$ and $d$ are parameter of the system. The chaotic behavior of the Lorenz hyperchaotic system is shown in Fig. 6, with initial values for the system state variables as $x_{1}(0)=, x_{2}(0)=, x_{3}(0)=$ and $x_{4}(0)=$ and the system parameters as $\alpha_{1}=36, \alpha_{2}=3, \alpha_{3}=20$ and $\alpha_{4}=1.3$

The leader system can be defined based on the Chen hyperchaotic system (16) as follows:

$$
\begin{aligned}
& x_{1}=a\left(x_{2}-x_{1}\right)+x_{4}+\eta_{1} \\
& x_{2}=d x_{1}+c x_{2}-x_{1} x_{3}+\eta_{2} \\
& x_{3}=x_{1} x_{2}-b x_{3}+\eta_{3} \\
& x_{4}=x_{1} x_{2}+r x_{4}+\eta_{4}
\end{aligned}
$$

Where $\eta_{1}, \eta_{2}, \eta_{3}$ and $\eta_{4}$ are the noise disturbances of the system. Then, consider the Lorenz hyperchaotic system (17), as the follower system as follows:

$$
\begin{aligned}
& y_{1}=a\left(y_{2}-y_{1}\right)+y_{4}+u_{1} \\
& y_{2}=-y_{1} y_{3}+d y_{1}-y_{2}+u_{2} \\
& y_{3}=y_{1} y_{2}-b y_{3}+u_{3} \\
& y_{4}=-y_{1} y_{3}+c y_{4}+u_{4}
\end{aligned}
$$


Where $u_{1}, u_{2}, u_{3}$ and $u_{4}$ are the feedback controller of the system.

The proposed chaos synchronization between the leader Chen hyperchaotic System (18) and the follower Lorenz hyperchaotic system (19) can be achived by designing an appropriate control law and noise estimation law as follows:

$$
\begin{aligned}
& u_{1}=-a y_{2}+\lambda_{1} a x_{1}+a e_{1}-e_{4}+\lambda_{1} \bar{\eta}_{1}-k_{1} e_{1} \\
& u_{2}=+y_{1} y_{3}-\lambda_{2} x_{1} x_{3}-d y_{1}+\lambda_{2} d x_{1}+y_{2}+c \lambda_{2} x_{2}+\lambda_{2} \bar{\eta}_{2}-k_{2} e_{2} \\
& u_{3}=-y_{1} y_{2}+\lambda_{3} x_{1} x_{2}+b e_{3}+\lambda_{3} \bar{\eta}_{3}-k_{3} e_{3} \\
& u_{4}=y_{1} y_{3}+\lambda_{4} x_{1} x_{2}-c y_{4}+\lambda_{4} r x_{4}+\lambda_{4} \bar{\eta}_{4}-k_{4} e_{4},
\end{aligned}
$$

and,

$$
\begin{aligned}
& \dot{\bar{\eta}}_{1}=-\lambda_{1} e_{1}-\psi_{1}\left(\bar{\eta}_{1}-\eta_{1}\right) \\
& \dot{\bar{\eta}}_{2}=-\lambda_{2} e_{2}-\psi_{2}\left(\bar{\eta}_{2}-\eta_{2}\right) \\
& \dot{\bar{\eta}}_{3}=-\lambda_{3} e_{3}-\psi_{3}\left(\bar{\eta}_{3}-\eta_{3}\right) \\
& \dot{\bar{\eta}}_{4}=-\lambda_{4} e_{4}-\psi_{4}\left(\bar{\eta}_{4}-\eta_{4}\right)
\end{aligned}
$$

Now, some numerical results related to the proposed synchronization of two hyperchaotic systems are given. Consider the parameter of the leaer Chen hyperchaotic system (18) as $a=35, b=3, c=12, d=7$ and $r=0.5$ and its initial values are taken as, $x_{1}(0)=11, x_{2}(0)=5, x_{3}(0)=9$, and, $x_{4}(0)=13$ . In additiion, the initial values of the response Lorenz hyperchaotic system (19) are selected as: $y_{1}(0)=$ $1, y_{2}(0)=11, y_{3}(0)=2$ and $y_{4}(0)=3$. Consider the nosie disturbance values as $\eta_{1}=0.8, \eta_{2}=0.6, \eta_{3}=0.3$ and $\eta_{4}=0.5$. Let the gain constants as $k_{1}=2, k_{2}=2, k_{3}=2, k_{4}=2, \phi_{1}=1.5, \phi_{2}=1.5, \phi_{3}=1.5$ and $\phi_{4}=1.5$.

The effectiveness of the synchronization method for the contorling behavior of the Lorenz hyperchaotic system (19) to track the motion trajectories of the Chen hyperchaotic system (18) and the noise disturbance estimation are illustrated in Figure 3 and 4, respectively. Figure 3 shows that the state variables of the system (19) track effectively the motion trajectories of the leader chaotic system(18). In addition, in Figure 4 exhibit that the distance between noise disturbance and its estimation values converge to zero.
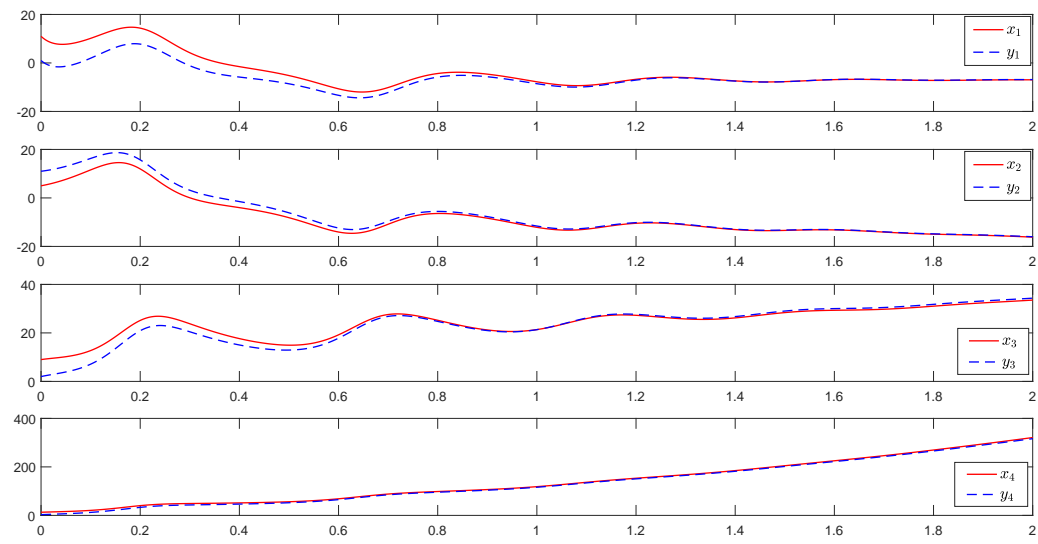

Figure 5. Time responce of the drive Zhang chaotic system and the response Lorenz chaotic system

\section{CONCLUSION}

In this research, some results related to the modified projective synchronization of known chaotic/hyperchaotic systems with noise disturbances are derived. Since the paramers of the leader system is considered knonwn, an appropriated active nonlinear feedback control law with designed via modified projective synchronization error. The validity of the proposed method is proved by means of Lyapunov stability theorem. Furtheremore, 


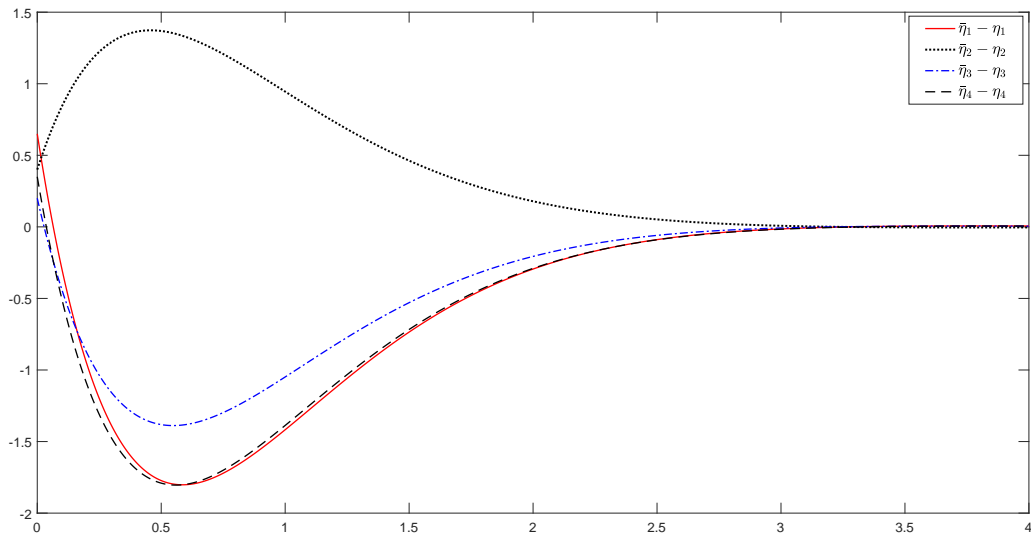

Figure 6. Time responce of the noise disturbance estimation

its effectiveness is verified by some numerical simulations of the chaotic and hyperchaotic systems. Finally, some figures are shown to verify the accuracy of the theorical discussions. As it can be seen from these results, the motion trajectories of the leader chaotic systems containing noise disturbances can effectively track by the state variables of the follower chaotic systems state variabels, which affected by proposed control method.

\section{REFERENCES}

[1] G. Chen, X. Yu, Chaos control: theory and applications, Vol. 292, Springer Science \& Business Media, 2003.

[2] C.-M. Lin, M.-H. Lin, R.-G. Yeh, Synchronization of unified chaotic system via adaptive wavelet cerebellar model articulation controller, Neural Computing and Applications 23 (3-4) (2013) 965-973.

[3] M.-c. Ho, Y.-C. Hung, Synchronization of two different systems by using generalized active control, Physics Letters A 301 (5) (2002) 424-428.

[4] E. A. Umoh, Adaptive Hybrid Synchronization of Lorenz-84 System with Uncertain Parameters, TELKOMNIKA Indonesian Journal of Electrical Engineering, vol.12, no.7, pp. 5251-5260, 2014

[5] H. Agiza, M. Yassen, Synchronization of rossler and chen chaotic dynamical systems using active control, Physics Letters A 278 (4) (2001) 191-197.

[6] G. M. Mahmoud, T. Bountis, E. E. Mahmoud, Active control and global synchronization of the complex chen and lü systems, International Journal of Bifurcation and Chaos 17 (12) (2007) 4295-4308.

[7] J. H. Park, Adaptive synchronization of hyperchaotic chen system with uncertain parameters, Chaos, Solitons \& Fractals 26 (3) (2005) 959-964.

[8] G. Zhang, Z. Liu, J. Zhang, Adaptive synchronization of a class of continuous chaotic systems with uncertain parameters, Physics Letters A 372 (4) (2008) 447-450.

[9] C. Zhu, Adaptive synchronization of two novel different hyperchaotic systems with partly uncertain parameters, Applied Mathematics and Computation 215 (2) (2009) 557-561.

[10] M. Rafikov, J. M. Balthazar, On control and synchronization in chaotic and hyperchaotic systems via linear feedback control, Communications in Nonlinear Science and Numerical Simulation 13 (7) (2008) 1246-1255.

[11] C. Li, X. Liao, Anti-synchronization of a class of coupled chaotic systems via linear feedback control, International Journal of Bifurcation and Chaos 16 (04) (2006) 1041-1047.

[12] L. Huang, R. Feng, M. Wang, Synchronization of chaotic systems via nonlinear control, Physics Letters A 320 (4) (2004) 271-275.

[13] H. Trabelsi, M. Benrejeb, Robust Control of the Unified Chaotic System. International Journal of Electrical and Computer Engineering. 2015 Feb 1;5(1):102.

[14] Q. Zhang, J.-a. Lu, Chaos synchronization of a new chaotic system via nonlinear control, Chaos, Solitons \& Fractals 37 (1) (2008) 175-179.

[15] M. Chen, Z. Han, Controlling and synchronizing chaotic genesio system via nonlinear feedback control, 
Chaos, Solitons \& Fractals 17 (4) (2003) 709-716.

[16] G. Ablay, Sliding mode control of uncertain unified chaotic systems, Nonlinear Analysis: Hybrid Systems 3 (4) (2009) 531-535.

[17] M. Yan, X. Zheng and J. Zhen, Synchronization of Hyperchaotic Systems under Active Adaptive Sliding Mode Control, TELKOMNIKA Indonesian Journal of Electrical Engineering, vol.11, no.11, pp. 67286736, 2013.

[18] M. S. Tavazoei, M. Haeri, Determination of active sliding mode controller parameters in synchronizing different chaotic systems, Chaos, Solitons \& Fractals 32 (2) (2007) 583-591.

[19] X. Yang, J. Cao, Hybrid adaptive and impulsive synchronization of uncertain complex networks with delays and general uncertain perturbations, Applied Mathematics and Computation 227 (2014) 480-493.

[20] M. G. Rosenblum, A. S. Pikovsky, J. Kurths, Phase synchronization of chaotic oscillators, Physical review letters 76 (11) (1996) 1804.

[21] Y.-W. Wang, Z.-H. Guan, Generalized synchronization of continuous chaotic system, Chaos, Solitons \& Fractals 27 (1) (2006) 97-101.

[22] C.-F. Feng, Projective synchronization between two different time-delayed chaotic systems using active control approach, Nonlinear Dynamics 62 (1-2) (2010) 453-459.

[23] R. Mainieri, J. Rehacek, Projective synchronization in three-dimensional chaotic systems, Physical Review Letters 82 (15) (1999) 3042.

[24] M. Hu, Z. Xu, Y. Yang, Projective cluster synchronization in drive-response dynamical networks, Physica A: Statistical Mechanics and its Applications 387 (14) (2008) 3759-3768.

[25] X. Wu, H. Lu, Projective lag synchronization of the general complex dynamical networks with distinct nodes, Communications in Nonlinear Science and Numerical Simulation 17 (11) (2012) 4417-4429.

[26] L. Runzi, D. Shucheng, W. Zhengmin, Modified projective synchronization of a hyperchaotic system with unknown and/or uncertain parameters, Journal of Vibration and Control (2010) 1077546310376988.

[27] H. Tirandaz, A. Hajipour, Adaptive synchronization and anti-synchronization of tsucs and lü unified chaotic systems with unknown parameters, Optik-International Journal for Light and Electron Optics 130 (2017) 543-549.

[28] J. Liu, S. Liu, C. Yuan, Adaptive complex modified projective synchronization of complex chaotic (hyperchaotic) systems with uncertain complex parameters, Nonlinear Dynamics 79 (2) (2015) 1035-1047.

[29] A. Al-Jarrah, M. Ababneh, S. B. Hani, K. Al-Widyan, Synchronization of chaotic systems with uncertain time-varying parameters, International Review of Mechanical Engineering (IREME) 9 (6) (2015) 568575 .

[30] H. Du, Q. Zeng, C. Wang, Modified function projective synchronization of chaotic system, Chaos, Solitons \& Fractals 42 (4) (2009) 2399-2404.

[31] X. Wu, J. Wang, Adaptive generalized function projective synchronization of uncertain chaotic complex systems, Nonlinear Dynamics 73 (3) (2013) 1455-1467.

[32] H. Kheiri, V. Vafaei, M. Moghaddam, Adaptive generalized function projective synchronization of uncertain hyperchaotic systems, International Journal of Nonlinear Science 14 (4) (2012) 434-442.

[33] L. Chao, W. Xingyuan, Modified function projective lag synchronization in fractional-order chaotic (hyperchaotic) systems, Journal of Vibration and Control (2013) 1077546312472921.

[34] S. Wang, S. Zheng, B. Zhang, H. Cao, Modified function projective lag synchronization of uncertain complex networks with time-varying coupling strength, Optik-International Journal for Light and Electron Optics 127 (11) (2016) 4716-4725.

[35] M. Yassen, Controlling chaos and synchronization for new chaotic system using linear feedback control, Chaos, Solitons \& Fractals 26 (3) (2005) 913-920.

[36] S. Bhalekar, V. Daftardar-Gejji, Synchronization of different fractional order chaotic systems using active control, Communications in Nonlinear Science and Numerical Simulation 15 (11) (2010) 3536-3546.

[37] J. Lü, G. Chen, A new chaotic attractor coined, International Journal of Bifurcation and chaos 12 (03) (2002) 659-661.

[38] Y. Li, W. K. Tang, G. Chen, Generating hyperchaos via state feedback control, International Journal of Bifurcation and Chaos 15 (10) (2005) 3367-3375.

[39] Q. Jia, Hyperchaos generated from the lorenz chaotic system and its control, Physics Letters A 366 (3) (2007) 217-222. 


\section{BIOGRAPHIES OF AUTHORS}

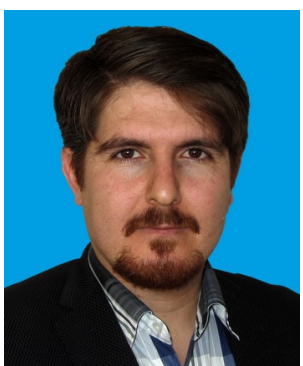

Hamed Tirandaz received the B.Eng. Degree in Applied Mathematics from the University of Sabzevar Tarbiat Moallem University, Sabzevar, Iran, in 2006, and PhD. degree in Mechatronics Engineering from Semnan University, Semnan, Iran, in 2009. He is been working as a Lecturer at Hakim Sabzevari University since 2010. His research interests include mainly Chaos control and synchronization. He has published several papers in the above mentioned area.

Email: tirandaz@hsu.ac.ir

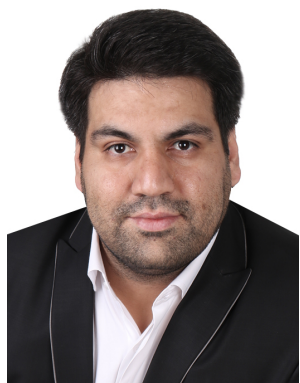

Mohsen Ahmadnia has received his PhD degree in Power Engineering at the People's Friendship university of Russia. He is currently with the Faculty of Electrical and Computer Engineering at Hakim Sabzevari University, Sabzevar, Iran. His research interests are in nonlinear dynamics.

Email: m.ahmadnia@hsu.ac.ir

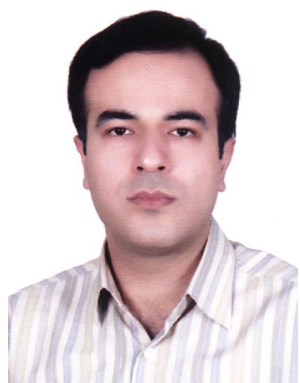

Hamidreza Tavakoli has obtained his $\mathrm{PhD}$ degree in Electrical Engineering at the Iran University of Science and Technology and Ryerson University in Toronto, Canada. He is currently with the Faculty of Electrical and Computer Engineering at Hakim Sabzevari University, Sabzevar, Iran. His research interests are in Chaos and nonlinear dynamics.

Email: tavakoli@hsu.ac.ir 